\title{
BMJ open Can the London 2012 Olympics 'inspire a generation' to do more physical or sporting activities? An overview of systematic reviews
}

\author{
Kamal Ram Mahtani, ${ }^{1,3}$ Joanne Protheroe, ${ }^{1,4}$ Sarah Patricia Slight, ${ }^{1,5,6}$ \\ Marcelo Marcos Piva Demarzo, ${ }^{1,7}$ Thomas Blakeman,, ${ }^{1,8}$ Christopher A Barton, ${ }^{1,9}$ \\ Bianca Brijnath, ${ }^{1,10}$ Nia Roberts ${ }^{2}$
}

To cite: Mahtani KR, Protheroe J, Slight SP, et al. Can the London 2012 Olympics 'inspire a generation' to do more physical or sporting activities? An overview of systematic reviews. BMJ Open 2013;3:e002058. doi:10.1136/bmjopen-2012002058

- Prepublication history for this paper are available online. To view these files please visit the journal online (http://dx.doi.org/10.1136/ bmjopen-2012-002058).

Received 4 September 2012 Revised 3 December 2012 Accepted 11 December 2012

This final article is available for use under the terms of the Creative Commons Attribution Non-Commercial 2.0 Licence; see http://bmjopen.bmj.com

For numbered affiliations see end of article.

Correspondence to Kamal R Mahtani; kamal.mahtani@phc.ox.ac.uk

\section{ABSTRACT}

Objective: To examine if there is an increased participation in physical or sporting activities following an Olympic or Paralympic games.

Design: Overview of systematic reviews.

Methods: We searched the Medline, Embase, Cochrane, DARE, SportDISCUS and Web of Knowledge databases. In addition, we searched for 'grey literature' in Google, Google scholar and on the International Olympic Committee websites. We restricted our search to those reviews published in English. We used the AMSTAR tool to assess the methodological quality of those systematic reviews included.

Primary and secondary outcome measures: The primary outcome was evidence for an increased participation in physical or sporting activities. Secondary outcomes included public perceptions of sport during and after an Olympic games, barriers to increased sports participation and any other nonsporting health benefits.

Results: Our systematic search revealed 844 citations, of which only two matched our inclusion criteria. The quality of these two reviews was assessed by three independent reviewers as 'good' using the AMSTAR tool for quality appraisal. Both reviews reported little evidence of an increased uptake of sporting activity following an Olympic Games event. Other effects on health, for example, changes in hospital admissions, suicide rates and drug use, were cited although there was insufficient evidence to see an overall effect.

Conclusion: There is a paucity of evidence to support the notion that hosting an Olympic games leads to an increased participation in physical or sporting activities for host countries. We also found little evidence to suggest other health benefits. We conclude that the true success of these and future games should be evaluated by high-quality, evidence-based studies that have been commissioned before, during and following the completion of the event. Only then can the true success and legacy of the games be established.

\section{ARTICLE SUMMARY}

Article focus

- Increased levels of physical activity are linked with improved health and may play a key role in the prevention or treatment of most noncommunicable diseases.

- The London 2012 Olympic and Paralympic games aims to leave a long-term legacy, which includes population level increases in physical and sporting activity.

- We conducted a systematic review of systematic reviews to establish whether hosting an Olympic games leads to increased participation in such activities.

Key messages

- There is little evidence that international elite sporting events such as the Olympics leads to increased participation in physical or sporting activities at the population health level. We found no evidence, in particular, relating to the Paralympic games.

- High-quality, evidence-based studies are needed to measure the true impact of the London 2012 games.

Strengths and limitations of this study

- This is a systematic review of existing systematic reviews.

- We restricted our search to those reviews published in English on previous Olympic and Paralympic games.

\section{INTRODUCTION}

The cost of hosting the 2012 Games of the XXX Olympiad in London has been estimated to be over $£ 8$ billion (UK) pounds. ${ }^{1}$ Part of the justification for spending this amount and bidding to host the games, comes from the belief that the event will leave a legacy in the UK. The theme of the games, 'Inspire a generation', reflected this 
desire to promote participation in sport in the run-up to, during and after the event. ${ }^{2}$

In 2010, the Department for Culture, Media and Sport outlined the UK Government's plans for this legacy, and included 'harnessing the UK's passion for sport to increase grass roots participation, particularly by young people-and to encourage the whole population to be more physically active'. ${ }^{3}$ However, since the games were awarded to London in 2005, there has been an overall decline among 16-year-olds to 25-year-olds in sport participation. ${ }^{4}$ These findings are a cause for concern especially as a recent analysis of the burden of disease and life expectancy showed that physical inactivity has a major negative health effect worldwide linked to coronary heart disease, diabetes and cancer. It is also estimated that around 1.3 million deaths could be averted every year if physical inactivity decreased by $25 \% .^{5}$

A previous systematic review of literature published between 1978 and 2008 found insufficient evidence to support or refute any health or socioeconomic impacts from major multisport events. ${ }^{6}$ We saw value in conducting an 'overview of systematic reviews', thus allowing the findings of separate reviews to be compared and contrasted. ${ }^{7}$ Second, we restricted our search to the impact of the Olympic and Paralympic games only, with our primary outcome of interest an increased participation in sport or recreational activities subsequent to hosting these games. Our aim was to highlight new areas that could possibly guide policy makers on decision making and planning of future games.

\section{METHODS}

Data sources and search strategy

Two authors (NR and KRM) devised the search strategy. One author (NR) searched MEDLINE, EMBASE, Cochrane, DARE, SportDISCUS and Web of Knowledge databases. For each database, we applied two search filters for identifying systematic reviews. ${ }^{8}$ We also performed searches on Google, Google Scholar and the International Olympic Committee websites. We restricted the inclusion of papers to those published in English (full details of the search strategy used are given in appendix 1).

\section{Study selection}

Three authors (KRM, JP and SPS) independently screened the results of the searches, according to the inclusion criteria, which were agreed a priori. Any disagreement between these authors was resolved by discussion. Given the broad nature of non-systematic reviews, commentaries and general opinion based articles, we restricted our search to only systematic reviews. We used the Cochrane Handbook for Systematic Reviews of Interventions for a definition of a systematic review, that is, 'a systematic review attempts to identify, appraise and synthesise all the empirical evidence that meets prespecified eligibility criteria to answer a given research question'. ${ }^{9}$ We included reviews which systematically appraised the published literature within the remit of the review. No restriction was placed on age, gender or race. We included reviews from all countries, and accepted reviews that presented their results quantitatively or as a narrative. We excluded reviews that only focused on other multisporting events (such as Winter Olympics and Commonwealth Games) as well as single-sporting events (such as World cups and World Championships).

\section{Outcomes}

The primary outcome for this overview was an increased involvement in sporting activity following an Olympic games. We define 'increased uptake' as any means to demonstrate increased participation, and held no restriction on whether this was demonstrated qualitatively or quantitatively. We applied the term 'sporting activity' to include any of the 36 Olympic sports (Archery, Athletics (including walking), Badminton, Basketball, Beach Volleyball, Boxing, Canoe Slalom, Canoe Sprint, Cycling-BMX, Cycling-Mountain Bike, Cycling — Road, Cycling-Track, Diving, Equestrian, Fencing, Football, GymnasticsArtistic, Gymnastics-Rhythmic, Handball, Hockey, Judo, Modern Pentathlon, Rowing, Sailing, Shooting, Swimming, Synchronised Swimming, Table Tennis, Taekwondo, Tennis, Trampoline, Triathlon, Volleyball, Water Polo, Weightlifting and Wrestling) and 21 Paralympic sports (Archery, Athletics, Boccia, Cycling Road, Cycling Track, Football 5-a-side, Football 7-a-side, Goalball, Equestrian, Judo, Powerlifting, Rowing, Sailing, Shooting, Swimming, Table Tennis, Sitting Volleyball, Wheelchair Basketball, Wheelchair Fencing, Wheelchair Rugby and Wheelchair Tennis).

Secondary outcomes included:

- Increases in other forms of physical activity;

- Public perceptions of sport during and after an Olympic games;

- Barriers to increased sports participation and

- Non-sporting (physical or mental) health benefits.

We followed the WHO definition of 'physical activity', that is, any bodily movement produced by skeletal muscles that requires energy expenditure. ${ }^{10}$ This was to include other forms of physical activity to include those sports not mentioned above as well as non-Olympic physical activities, for example, dance, skipping, etc.

\section{Quality assessment of included reviews}

We used the AMSTAR measurement tool to assess the quality of the included reviews. This 11-point assessment tool is, to the best of our knowledge, the only one validated tool for this purpose. ${ }^{11}$ The assessment was carried out independently by two authors (CB and $\mathrm{BB}$ ). Any disagreement between authors was referred to a third author (KRM) and a final decision was made.

\section{Data extraction}

Three authors (KRM, TB and MD) independently extracted data from included reviews using a predefined 
data extraction sheet. We included the title, author, year and funding source, study aim, search strategy, number of included studies, inclusion and exclusion criteria, population(s) for which the studies have been set in, Olympic games being referred to, overall conclusions and implications for future practice. We avoided looking at the primary data unless we felt further relevant information was needed.

\section{Review synthesis}

All extracted data were tabulated according to study ID, year of publication, AMSTAR quality score, data relating to our primary outcome and data relating to secondary outcome. The sections relating to our primary outcome were further divided into the Olympic event to which they refer the sporting activity and the overall impact. Quantitative synthesis was not possible and as a result it was agreed that all results be reported as a narrative.

\section{RESULTS}

\section{Results of search strategy}

Figure 1 summarises the results of our search strategy. We initially identified 844 references after removal of duplicates. Of these, 798 references were excluded based on titles alone for a lack of relevance. Of the

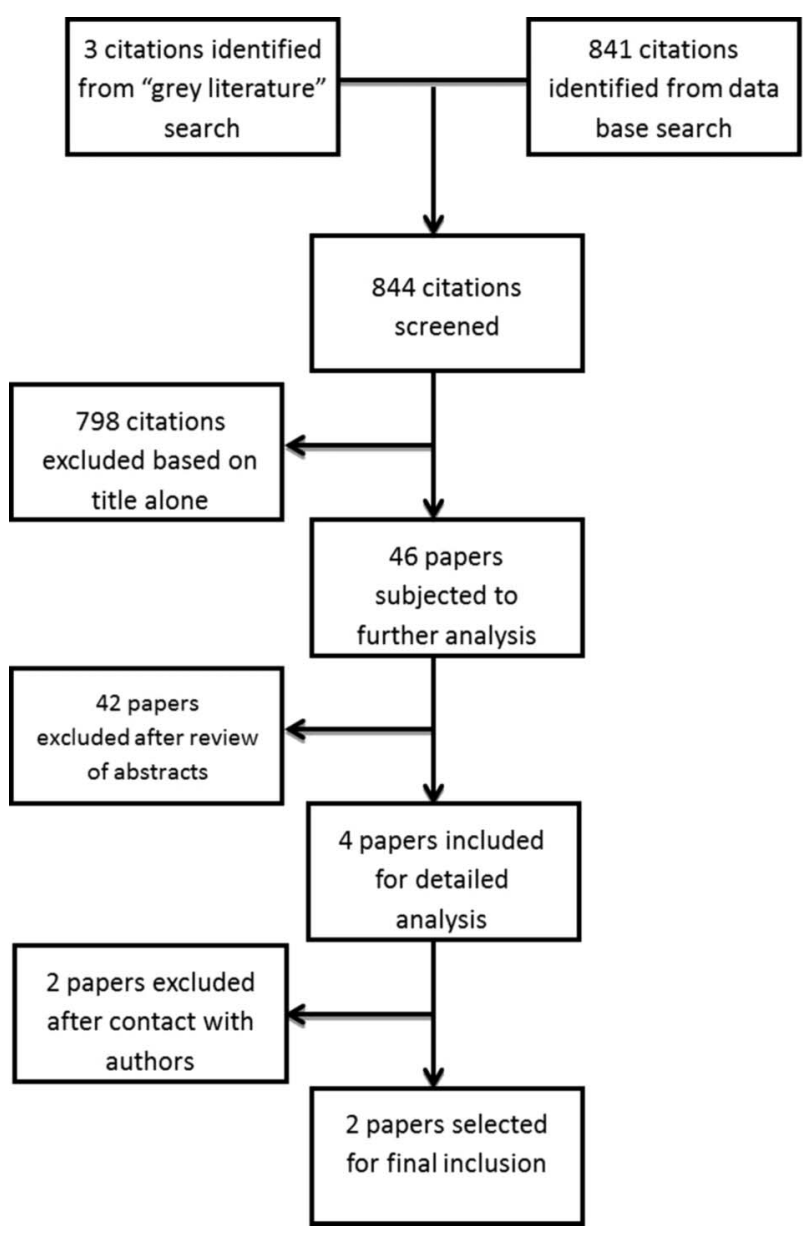

Figure 1 PRISMA flow diagram. remaining 46 papers, a further 42 were dismissed after review of the abstracts. The majority of excluded citations were non-systematic reviews, commentaries and books. Where possible, relevant chapters of books were reviewed and included if they met our criteria. Four reviews were analysed in detail. Of these, two were subsequently excluded. Although one of these excluded reviews searched key databases, specialist bibliographic databases and different types of evidence including the grey literature, as well as contacted relevant individuals and organisations, we were unclear if its review process was carried out systematically. ${ }^{12}$ We contacted the authors of this paper who confirmed that their study was not a systematic review and as a result it was excluded from our review(Murphy NM. personal communication (via email), 17 August 2012) The other excluded study ${ }^{13}$ was an abbreviated version of one of our included reviews. ${ }^{14}$ Personal communication with the lead author of both papers confirmed that the abbreviated version contained no additional information(Weed $\mathrm{M}$ personal communication (via email), 15 August 2012). As a result we excluded it from our review. It was finally agreed that only two reviews were eligible for quality assessment and data extraction. The first of these was a systematic review of the evidence base for developing a physical activity and health legacy from the London 2012 Olympic and Paralympic games, commissioned by the UK Department of Health. ${ }^{14}$ The second included study was a systematic review evaluating the health and socioeconomic impacts of major multisporting events between 1978 and 2008. ${ }^{6}$ Table 1 summarises the main characteristics from the included reviews.

\section{Quality assessment of included reviews}

We only selected reviews where the objective of the study was clearly stated and fitted within the remit of this review. We used the AMSTAR methodological quality assessment tool to appraise our included reviews. ${ }^{11}$ Overall, we found the quality of the included reviews to be good. Table 2 summarises the results of our quality appraisal using the AMSTAR tool.

\section{Increased uptake of sporting or physical activity following} an Olympic games

Both included reviews reported that the evidence to support an uptake of sport or physical activities after an Olympic games was generally weak and inconclusive. McCartney et at reported no overall change in the recreational impact of hosting an Olympic games. They cited some evidence, however, of an upward trend in sports participation from the early 1980s until 1994, and in association with the 1992 Barcelona games in Spain. They graded the cited evidence as being of the lowest level according to their quality appraisal. Weed et $a l^{14}$ also referred to evidence for increased participation following the 1992 Barcelona games. The authors noted that the results were drawn from data taken 7 years prior and 3 years after the 1992 games, and highlighted a 
Table 1 Characteristics of included reviews

\begin{tabular}{|c|c|c|}
\hline Review & Weed et $a I^{14}$ & McCartney et $a{ }^{6}$ \\
\hline Year & 2009 & 2010 \\
\hline $\begin{array}{l}\text { Question posed by } \\
\text { review }\end{array}$ & $\begin{array}{l}\text { Four questions were established for the review: } \\
\text { (1) What evidence exists that the Olympic } \\
\text { Games, sports events or sports franchises can } \\
\text { impact upon physical activity and sport } \\
\text { participation and upon health-related } \\
\text { behaviours? } \\
\text { (2) By what processes have physical activity and } \\
\text { sport participation and health-related behaviours } \\
\text { been leveraged from the Olympic Games, sports } \\
\text { events or sports franchises? } \\
\text { (3) What processes that have been used to } \\
\text { leverage, inter alia, volunteering, community } \\
\text { engagement and tourism from the Olympic } \\
\text { Games, sports events and sports franchises } \\
\text { might inform leveraging strategies for physical } \\
\text { activity, sport and health? } \\
\text { (4) How has the leveraging of a range of } \\
\text { opportunities from Olympic Games, sports } \\
\text { events and sports franchises been evaluated? }\end{array}$ & $\begin{array}{l}\text { To assess the effects of major multisport events } \\
\text { on health and socioeconomic determinants of } \\
\text { health in the population of the city hosting the } \\
\text { event }\end{array}$ \\
\hline Search strategy & $\begin{array}{l}\text { Published literature via SPORTS DISCUS , } \\
\text { CINAHL, PsychINFO, MEDLINE, } \\
\text { Web of Knowledge (General Science and } \\
\text { Social Science Database) } \\
\text { In addition several sources for 'grey literature' } \\
\text { were searched (see full paper for more details) }\end{array}$ & $\begin{array}{l}\text { Papers published between } 1978 \text { and } 2008 \\
\text { From Applied Social Science Index and } \\
\text { Abstracts (ASSIA), British Humanities Index } \\
\text { (BHI), Cochrane database of systematic reviews, } \\
\text { Econlit database, Embase, Education Resources } \\
\text { Information Center (ERIC)database, Health } \\
\text { Management Information Consortium (HMIC) } \\
\text { database, International Bibliography of the Social } \\
\text { Sciences (IBSS), Medline, PreMedline, } \\
\text { PsycINFO, Sociological Abstracts, Sportdiscus, } \\
\text { Web of Knowledge, Worldwide Political Science } \\
\text { Abstracts } \\
\text { In addition an 'extensive' search of the grey } \\
\text { literature (between April \& October 2008) was } \\
\text { carried out (see individual review for full details) }\end{array}$ \\
\hline No of included studies & 24 & 54 \\
\hline $\begin{array}{l}\text { Quality appraisal tool } \\
\text { used to assess } \\
\text { included studies }\end{array}$ & $\begin{array}{l}\text { A rudimentary quality appraisal sheet was } \\
\text { agreed by all authors and review panel as being } \\
\text { relevant to the research question (Weed } \\
\text { M. personal communication (via email), } 15 \\
\text { August 2012) }\end{array}$ & $\begin{array}{l}\text { Assessed using a modified version of the } \\
\text { Hamilton quality assessment tool }\end{array}$ \\
\hline $\begin{array}{l}\text { Overall comment on } \\
\text { quality of included } \\
\text { studies }\end{array}$ & $\begin{array}{l}\text { Variable. With reference to our primary outcome, } \\
\text { was assessed to be generally poor }\end{array}$ & Study quality was 'poor' \\
\hline
\end{tabular}

possible limitation in comparing data taken at different times and by different designs. The authors also concluded that the evidence was mixed for a 'trickle down' effect on participation; it was possible that some shortterm benefits may have been seen but little evidence of a long-term effect.

\section{Public perceptions to sporting activity}

\section{after an Olympic games}

McCartney et $a l^{6}$ did not cite any specific evidence of changes in the public's perception of sporting or physical activity following an Olympic games. In contrast,
Weed et $a l^{14}$ made reference to a positive perception following elite sporting success. However, they also cited evidence for a negative effect with the potential of elite sports deterring individual participation because of a perceived competence gap. The review also cited evidence for a lack of increased physical activity following the 2000 Sydney Olympics in Australia, despite the 'euphoria' that was documented as following the Games. Weed et al also made reference to evidence from Sport England (UK) that found "more than a quarter of the population in England (26\%) have been inspired by British medal-winning performances at the Olympic 


\begin{tabular}{|c|c|c|c|}
\hline & Question & Weed et $a l^{14}$ & McCartney et $a^{\rho}$ \\
\hline 1. & Was an 'a priori' design provided? & $\mathrm{Y}$ & Y \\
\hline 2. & Was there duplicate study selection and data extraction? & Y & $\mathrm{Y}$ \\
\hline 3. & Was a comprehensive literature search performed? & $\mathrm{Y}$ & $\mathrm{Y}$ \\
\hline 4. & $\begin{array}{l}\text { Was the status of publication (ie, grey literature) used as } \\
\text { an inclusion criterion? }\end{array}$ & Y & Y \\
\hline 5. & Was a list of studies (included and excluded) provided? & $\mathrm{N}$-only included studies & $\mathrm{N}$-only included studies \\
\hline 6. & $\begin{array}{l}\text { Were the characteristics of the included studies } \\
\text { provided? }\end{array}$ & $\mathrm{Y}$ & $\mathrm{Y}$ \\
\hline 7. & $\begin{array}{l}\text { Was the scientific quality of the included studies } \\
\text { assessed and documented? }\end{array}$ & Y & Y \\
\hline 8. & $\begin{array}{l}\text { Was the scientific quality of the included studies used } \\
\text { appropriately in formulating conclusions? }\end{array}$ & Y & Y \\
\hline 9. & $\begin{array}{l}\text { Were the methods used to combine the findings of } \\
\text { studies appropriate? }\end{array}$ & Y & Y \\
\hline 10. & Was the likelihood of publication bias assessed & Y & $\begin{array}{l}\text { N/A-authors state this } \\
\text { not possible }\end{array}$ \\
\hline 11. & Was the conflict of interest stated? & $\begin{array}{l}U \text { (although commissioned by } \\
\text { Department of Health }\end{array}$ & Y \\
\hline & Total & 9 & 9 \\
\hline
\end{tabular}

Games in Athens (2004)'. However, they suggested that this was likely to be due to increased participation from people already partaking in sporting activity. They did however acknowledge the importance of an Olympic games in generating a 'festival effect' with evidence that such a perception has the potential to increase the desire to participate in all aspects of the games, including the increased uptake of physical activity.

\section{Barriers to partaking in increased sporting activity}

Weed $e t a l^{14}$ cite evidence that a negative perception to the games could act as a barrier or negate completely the potential to use such an event for the development of physical activity or sport, or the promotion of health. The authors also infer from their results, that the absence of planning supplemental activities to leverage and follow the main event, may also act as a barrier to further participation. Community and social empowerment were themes that were reported as being important facilitators in increasing sporting activity.

\section{Other health benefits}

The McCartney et at study examined a number of other outcomes relating to health, well-being, quality of life, health service use, as well as recreation. They found the quality of evidence to be mixed with no overall conclusion for a clear benefit. A moderate grade of evidence was ascribed to data relating to suicide rates which were found to be unchanged after the 1988 Seoul Olympics in South Korea. In contrast, lower levels of evidence were awarded to data showing an increase in paediatric health service demand, and a decrease in childhood asthma acute care events following the 1996 Olympics in Atlanta, USA. A low level of evidence was given to data relating to an increase in hospital presentations related to illicit drugs following the 2000 Olympics in Sydney, Australia. Weed $e a^{14}$ also report no overall clear benefit on health after an Olympic games event. They cite smoking cessation data, following a campaign to reduce smoking around the Barcelona Olympics that were inconclusive in showing a positive link. They also referred to data from China in the build-up to the 2008 Beijing Olympics suggesting that general health and extended life expectancy improved using data gained from a 'national physique examination'. However, Weed et al point out that such results should be interpreted with caution as it may not be possible to extrapolate them to other environments. A broader but similar point is discussed in the McCartney et al review who point out that 'both the commissioning of studies and their publication could well be biased towards positive results'.

\section{DISCUSSION}

Few systematic reviews have been published on the physical and sporting impact(s) that Olympic games can have on the public. To the best of our knowledge, this is the first systematic review of systematic reviews to assess whether an Olympic event leads to an increased uptake in physical or sporting activities, in addition to other health benefits.

Overall we found little evidence to support a clear positive impact either on the uptake of activities or on other health benefits linked to hosting an Olympic games. Our review suggests that a number of factors are likely to have contributed to this. First, it cannot be expected that such events will automatically increase activity simply by the event taking place. Instead, we found that a number of factors were likely to be needed, such as a 'positive' 
perception in advance of the games, the idea that participation in physical activity need not be limited to elite sportsmen, and that there will be sufficient infrastructure to access and partake in activities within the community and schools setting after the games. As pointed out by Weed $e t a l,{ }^{14}$ the generation of a 'festival' feel is likely to generate short-term positive perceptions and increased participation in a number of areas including sporting activity. We also noted that both included reviews eluded that collecting data on increased physical activity after such an event may be challenging, poor or absent. As a result, we recommend that evidence-based strategies to record this data, locally and regionally, should already be in place following the 2012 London Games. We further recommend that such methods are executed in advance and following the 2016 Olympic Games in Rio, Brazil.

We also noted that the London 2012 Olympic games, more so than previous ones, have placed a greater emphasis on leaving a legacy. As a result, pressure will mount on ensuring that this is achieved. We note that the 2010 Department for Culture Media and Sport document outlining the UK Governments plans for a legacy after the Olympic games included numerous proposals to improve mass participation in sport and increased activity. However, the government proposals from 2010 contrast with recent reports, following the 2012 games, of a decline in sports diversity and coaching as a result of funding cuts. ${ }^{15}$ Part of the problem may lie in the difficulty in measuring this impact. As pointed out in the 2010 editorial several confounders are likely to contribute to an apparent increase in sports participation. ${ }^{16}$ For example, a free swimming programme for under 16-year-olds and over 60-year-olds may simply result in people who already swim, now swimming for free or more often. This then does not meet the remit of facilitating more people to participate. ${ }^{16}$

\section{Limitations of our review}

We were aware of several limitations to our review. We chose to only include data relating to the summer Olympic and Paralympic games, thus excluding the Winter Olympics as we felt that the summer games would have a greater potential of encouraging events that could be transferable and available for mass participation worldwide. We also avoided including data from other multisporting events, such as the Commonwealth games, on the basis that no other single, multisporting, event had the same breadth of included sports or public interest as the Olympics. However, we note that data did exist, particularly following the 2002 Manchester Commonwealth games, but evidence of a definite link to increased activity was inconclusive. It was, therefore, unlikely to have changed the conclusions of our review.

We also limited our searching of 'grey literature' to Google, Google scholar and the International Olympic Committee websites, as we felt that there would be a significant number of non-systematic reviews and commentaries on other sites that would not meet our inclusion criteria.

\section{OVERALL CONCLUSION}

The evidence to support the notion that hosting an Olympic games leads to an automatic increase in mass sporting or physical activity is poor. Our review has found several areas of potential that could be capitalised on to test this hypothesis. Having existing routes into increased participation is likely to prove beneficial. An emphasis of involvement as well as targeting certain populations such as children and those contemplating activity, rather than just those already involved in it. The framing of an Olympic games in a broader sense, such as through a positive public perception and within a 'festival' feeling, is also likely to reap benefits. The UK will host the 2014 Glasgow Commonwealth games and has a unique opportunity to build on the public interest generated from the London 2012 games. ${ }^{17}$ We also suggest that in the interim, smaller events relating to increase sporting or physical activity, be put into place to keep the momentum generated from London 2012 going. Such events could then be capitalised on in the lead up to the 2016 Olympics in Brazil. The effects should be recorded using high-quality, evidencebased methods. Through such means the true success and legacy of the London 2012 games will be determined.

\section{Author affiliations}

${ }^{1}$ Members of the Brisbane Initiative (Cohort 7), International Leadership Programme in Primary Care, Oxford, UK

${ }^{2}$ Knowledge Centre, Bodleian Health Care Libraries, University of Oxford, Oxford, UK

${ }^{3}$ Department of Primary Health Care Sciences, University of Oxford, Oxford, UK ${ }^{4}$ Institute of Primary Care and Health Sciences, Arthritis Research UK Primary Care Centre, Keele University, Oxford, UK

${ }^{5}$ Division of Primary Care, The University of Nottingham, Nottingham, UK ${ }^{6}$ Division of General Internal Medicine, Brigham and Women's Hospital and Harvard Medical School, Boston, Massachusetts, USA

${ }^{7}$ General Practice Unit, Department of Preventive Medicine, Escola Paulista de Medicina, Universidade Federal de São Paulo (UNIFESP), Sao Paulo, Brazil

${ }^{8}$ Primary Care Research, Health Sciences Research Group, School of Community Based Medicine, University of Manchester, Manchester, UK

${ }^{9}$ Flinders Prevention, Promotion and Primary Health Care Cluster School of Medicine Flinders University Bedford Park, South Australia

${ }^{10}$ Department of General Practice, Monash University, Melbourne, Australia

Acknowledgements We are grateful to Professor Mike Clarke for his advice relating to the methodology section of this review. We also thank Dr Jeremy Howick for commenting on the original draft protocol and Meena Mahtani for proof reading the final draft.

Contributors KRM, JP, SPS, MD, TB, CB and BB are all members of Cohort 7 of The International Primary Care Research Leadership programme, part of The Brisbane Initiative to develop future leaders in primary care research. KRM had the initial idea for the review and drafted the first protocol. All authors commented and advised on this draft. KRM and NR devised the search strategy which was conducted by NR. KRM, JP and SPS screened the results of the searches and agreed on the final list of included reviews. $C B$ and $\mathrm{BB}$ assed the included reviews for quality. TB and MD carried out the data extraction. KRM wrote the first draft of the final papers with contributions and edits from all remaining authors. All authors contributed to the final draft.

Funding This research received no specific grant from any funding agency in the public, commercial or not-for-profit sectors. 
Competing interests None.

Provenance and peer review Not commissioned; externally peer reviewed.

Data sharing statement No additional data are available.

\section{REFERENCES}

1. Flyvbjerg B, Stewart A. Olympic proportions: cost and cost overrun at the Olympics 1960-2012, Working Paper: Saïd Business School, University of Oxford, 2012.

2. London 2012 Inspire programme. http://www.london2012.com/ about-us/inspire/inspire-programme/2012 (accessed 1 Aug 2012).

3. Plans for the legacy from the 2012 Olympic and paralymic games. In: Department for Culture, Media, and, Sport, editors. London, 2010: http://www.culture.gov.uk/publications/7674.aspx (accessed 1 Aug 2012).

4. Creating a sporting habit for life: a new youth sport strategy. In: Department for Culture, Media, and, Sport, editors, 2012.

5. Lee IM, Shiroma EJ, Lobelo F, et al. Effect of physical inactivity on major non-communicable diseases worldwide: an analysis of burden of disease and life expectancy. Lancet 2012;380:219-29.

6. McCartney G, Thomas S, Thomson H, et al. The health and socioeconomic impacts of major multi-sport events: systematic review (1978-2008). BMJ 2010;340:c2369.

7. Smith V, Devane D, Begley CM, et al. Methodology in conducting a systematic review of systematic reviews of healthcare interventions. BMC Med Res Methodol 2011;11:15.

8. ISSG Search filter resource. Systematic reviews: centre for reviews and dissemination. 2012. http://www.york.ac.uk/inst/crd/intertasc/sr. htm (accessed 9 Aug 2012).

9. Higgins JPT GSe. Cochrane handbook for systematic reviews of interventions. Cochrane Collab 2011; Version 5.1.0 (updated March 2011). http://www.cochrane-handbook.org (accessed 10 Aug 2012).

10. WHO. Health topics: physical activity. http://www.who.int/topics/ physical_activity/en/ (accesed 1 Aug 2012).

11. Shea BJ, Hamel C, Wells GA, et al. AMSTAR is a reliable and valid measurement tool to assess the methodological quality of systematic reviews. J Clin Epidemiol 2009;62:1013-20.

12. Murphy NM, Bauman A. Mass sporting and physical activity events-are they 'bread and circuses' or public health interventions to increase population levels of physical activity? J Phys Act Health 2007; 4:193-202.

13. Weed M, Coren E, Fiore J, et al. Developing a physical activity legacy from the London 2012 Olympic and Paralympic games: a policy-led systematic review. Perspect Public Health 2012;132:75-80.

14. Weed M, Coren E, Fiore J, et al. A systematic review of the evidence base for developing a physical activity and health legacy from the London 2012 Olympic and Paralympic games. London: Department of Health. 2009. http://www.london.nhs.uk/webfiles/Independent\% 20inquiries/Developing\%20physical\%20activity\%20and\%20health\% 20legacy\%20-\%20full\%20report.pdf (accessed 10 Aug 2012).

15. Campbell JVaD. Beyond the Olympic glory lies a patchy future for school sports. The Guardian Thursday 9 August 2012.

16. Weed M. How will we know if the London 2012 Olympics and Paralympics benefit health? BMJ 2010;340:c2202.

17. McCartney G, Palmer S, Winterbottom J, et al. A health impact assessment of the 2014 Commonwealth Games in Glasgow. Public Health 2010;124:444-51.

\section{APPENDIX 1}

\section{Search strategies}

MEDLINE (OvidSP) (1946_in process)—9 August 2012

1 (olympic* or paralympic ${ }^{\star}$ ).ti,ab. 2255

2 (sport* adj (event* or mega-event $\left.{ }^{\star}\right)$ ).ti,ab. 505

3 ((international or multination* or 15

multi-nation*) adj event* $^{\star}$.ti,ab. and sport* .

$\mathrm{mp}$.

4 ((international or multination* or

10

multi-nation $^{\star}$ ) adj games).ti,ab.

51 or 2 or 3 or 4

6 (Medline or systematic review).tw. or 87371 meta-analysis.pt.

75 and 6
8 meta-analysis.mp,pt. or review.pt. or

1884987 search:.tw.

95 and 8

358

EMBASE (OvidSP) (1974-)—9 August 2012

1 (olympic* or paralympic*).ti,ab. 2799

2 (sport $^{\star}$ adj (event ${ }^{\star}$ or mega-event $\left.{ }^{\star}\right)$ ).ti,ab. 649

3 ((international or multination* or multi-nation*) 23 adj event $\left.{ }^{\star}\right)$.ti,ab. and sport ${ }^{*} . \mathrm{mp}$.

4 ((international or multination* or multi-nation*) 17 adj games).ti,ab.

51 or 2 or 3 or 4

6 (meta-analysis or systematic review or 111218 MEDLINE).tw.

75 and 6

8 meta-analy*:.mp. or search*.tw. or review.pt.

95 and 8

Cochrane Database of Systematic Reviews \& Database of Abstracts of Reviews of Effectiveness (Cochrane Library, Wiley)8 August 2012

\#1 $\quad$ (olympic $^{\star}$ or paralympic* or special olympic ${ }^{\star}$ ):ti, 71 ab,kw

\#2 (sport ${ }^{\star}$ near $\left(\right.$ event $^{*}$ or mega-event $\left.\left.{ }^{\star}\right)\right): t i, a b, k w \quad 25$

\#3 (international or multination* or multi-nation*) 1 near event* and sport*:ti,ab,kw

\#4 ((international or multination* or multi-nation*) 1 near games):ti,ab,kw

SportDISCUS (EBSCOHost) (1980—)—9 August 2012

S9 S5 and S8

S8 TI (meta-analy* or review) OR AB

(485)

(meta-analy* or search*)

S7 S5 and S6

S6 TI (meta-analysis or systematic review or MEDLINE) OR AB (meta-analysis or systematic review or MEDLINE) OR KW (meta-analysis or systematic review or MEDLINE)

S5 S1 or S2 or S3 or S4

S4 TI (((international or multination* or

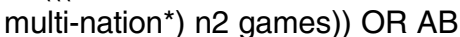

(((international or multination* or multi-nation*) n2 games))

S3 TI (((international or multination* or multi-nation $\left.^{\star}\right) \mathrm{n} 2$ event $\left.^{\star}\right)$ ) OR $A B$ (((international or multination* or multi-nation* ${ }^{\star}$ n2 event*))

S2 TI ((sport ${ }^{\star}$ n2 (event* or mega-event $\left.\left.\left.{ }^{\star}\right)\right)\right)$ OR AB ((sport* n2 (event* or mega-event $\left.\left.{ }^{\star}\right)\right)$ )

$\mathrm{TI}$ (olympic* or paralympic*) OR AB (olympic $^{*}$ or paralympic*)

Science Citation Index-EXPANDED, Social Science Citation Index (Web of Knowledge) (All years)

\# 468 Topic=(olympic* OR paralympic*) AND Topic $=$ (meta-analys* OR 'systematic review' OR 'evidence review' OR medline OR search*) \# 323 \#2 OR \#1 
\# 221 Title=(olympic* OR paralympic $\left.{ }^{\star}\right)$

AND Topic $=$ (meta-analys* OR 'systematic review' OR 'evidence review' OR medline OR search*)

\# 13 Topic=(olympic* OR paralympic*)

AND Title $=$ (meta-analys ${ }^{*}$ OR 'systematic review' OR 'evidence review')

Search filters used:

The validated search filters for MEDLINE and EMBASE were taken from the following publications. The search of Cochrane Library is self-limiting as we included references within Cochrane Database of Systematic Reviews (CDSR) \& Database of Abstracts of reviews of Effectiveness (DARE). There are no validated filters for SportsDISCUS or Web of Science-we applied keywords adapted from those used in the MEDLINE \& EMBASE searches for these two databases.
EMBASE search strategies achieved high sensitivity and specificity for retrieving methodologically sound systematic reviews. Wilczynski NL, Haynes RB; Hedges Team.

J Clin Epidemiol 2007;60:29-33.

Optimal search strategies for retrieving systematic reviews from Medline: analytical survey. Montori VM, Wilczynski NL, Morgan D, Haynes RB; Hedges Team.

BMJ 2005;330:68.

Search line $7=$ Best specificity

(Medline or systematic review).tw. or meta-analysis.pt.

99.2 (99.1 to 99.3)

Search line $9=$ Top strategy minimising the difference between sensitivity and specificity

meta-analysis.mp,pt. or review.pt or search:.tw.

Sensitivity reported as 98.0 (97.0 to 99.0)

- Excluded papers prior to 1987

- Excluded animal studies 